\title{
Effects of the Diabetic Condition on Grafted Fat Survival: An Experimental Study Using Streptozotocin-Induced Diabetic Rats
}

\author{
Jae A Jung ${ }^{1}$, Yang Woo Kim², Young Woo Cheon'2, So Ra Kang ${ }^{3}$ \\ ${ }^{1}$ Department of Plastic Surgery, Korea University Guro Hospital, Korea University College of Medicine, Seoul; ${ }^{2}$ Department of Plastic and \\ Reconstructive Surgery, Gachon University Gil Medical Center, Incheon; ${ }^{3}$ Department of Plastic and Reconstructive Surgery, Ewha Womans \\ University Hospital, Ewha Womans University School of Medicine, Seoul, Korea
}

Background Autologous fat grafts have been widely used for cosmetic purposes and for soft tissue contour reconstruction. Because diabetes mellitus is one of the major chronic diseases in nearly every country, the requirement for fat grafts in diabetes patients is expected to increase continuously. However, the circulation complications of diabetes are serious and have been shown to involve microvascular problems, impairing ischemia-driven neovascularization in particular. After injection, revascularization is vital to the survival of the grafted fat. In this study, the authors attempted to determine whether the diabetic condition inhibits the survival of injected fat due to impaired neovascularization.

Methods The rat scalp was used for testing fat graft survival. Forty-four seven-weekold male Sprague-Dawley rats were allocated to a diabetic group or a control group. 1.0 $\mathrm{mL}$ of processed fat was injected subcutaneously into the scalp of each rat. The effect of diabetes was evaluated by calculating the volume and the weight of the grafted fat and by histologically analyzing the fat sections.

Results The surviving fat graft volume and weight were considerably smaller in the diabetic group than in the control group $(\mathrm{P}<0.05)$, and histological evaluations showed less vascularity, and more cysts, vacuoles, and fibrosis in the diabetic group $(\mathrm{P}<0.05)$. Cellular integrity and inflammation were not considerably different in the two groups.

Conclusions As the final outcome, we found that the presence of diabetes might impair the survival and the quality of fat grafts, as evidenced by lower fat graft weights and volumes and poor histologic graft quality.

Keywords Diabetic angiopathies / Adipose tissue / Streptozocin
Correspondence: So Ra Kang Department of Plastic and Reconstructive Surgery, Ewha Womans University Hospital, Ewha Womans University School of Medicine, 1071 Anyangcheon-ro, Yangcheon-gu, Seoul 158-710, Korea Tel: +82-2-2650-5149

Fax: +82-2-2651-9821

E-mail: srps@ewha.ac.kr

\section{INTRODUCTION}

Autologous fat grafts are widely used for cosmetic purposes, for correcting soft tissue contour defects, and for reconstruction, mainly because of their straightforwardness and relatively few associated problems. The major drawback of this procedure is the high absorption rate of the injected fat, which can be $20 \%$ to $70 \%$ by volume $[1]$.
No potential conflict of interest relevant to this article was reported. 
On the fourth day after injection, new angiogenesis from the host vascular network causes graft revascularization [2], but this process is rather limited to the fat cell mass periphery and centrally located adipocytes have little contact with the vascular supply. Accordingly, the death and removal of the fat cells continue until the reorganized blood supply matches the graft vascular demands, and thus, early and appropriate revascularization is important for graft survival $[3,4]$.

Diabetes mellitus is one of the major chronic diseases in nearly every country. The universal prevalence of diabetes among adults (aged 20-79 years) was 6.4\% in 2010, and this percentage is expected to increase to $7.7 \%$ by 2030 [5]. Therefore, the number of diabetic candidates requiring an aesthetic fat graft will also gradually increase. However, the circulation complications of diabetes are serious and have been shown to involve microvascular problems, particularly with respect to the impairment of collateral vessel formation [6]. Furthermore, many experimental studies suggest that diabetes impairs ischemia-driven neovascularization.

The principal assumption of this study was that the diabetic condition inhibits the survival of the grafted fat by impairing neovascularization. To investigate this assumption, a rat scalp fat graft model was designed to evaluate the fat survival. The effect of the presence of diabetes on the grafted fat was estimated by calculating the volume and the weight of the grafted fat and by a performing histological analysis on the grafted fat sections.

\section{METHODS}

\section{Fat tissue preparation and processing}

The fat used in this study was harvested from a 35-year-old patient with no history of any underlying disease, who underwent reduction mammoplasty under general anesthesia. Written informed consent was procured before the operation. Approximately $40 \mathrm{~g}$ of adipose tissue was obtained (Fig. 1) and this was washed extensively with phosphate-buffered saline (Sigma-Aldrich, St. Louis, MO, USA) and cut into pieces of less than $1 \mathrm{~mm}$

\section{Fig. 1. A photograph of the excised donor fat}

Intraoperative photo of the excised donor fat.

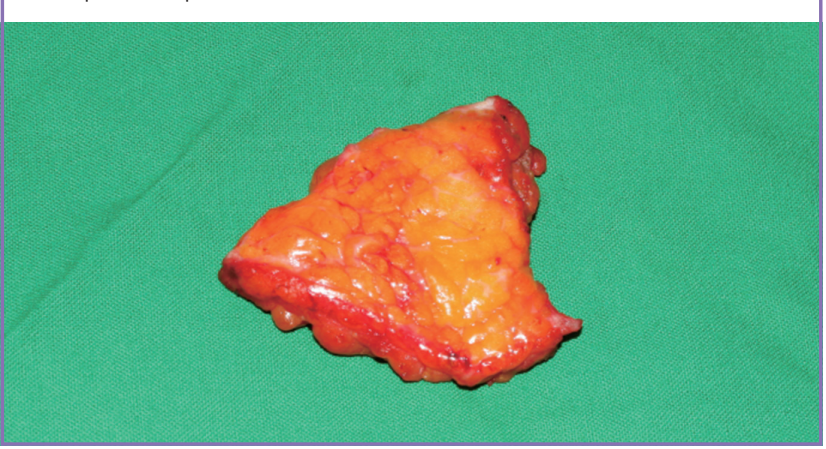

in all dimensions by using a no. 10 blade. The fat was processed under sterile conditions by placing it on an operating room sterile cotton towel, which allowed the absorption of fluids. During this process, the fat was exposed to air for almost 10 minutes. Plasma and cell debris were removed by centrifuging a fat suspension for 3 minutes at $3,000 \mathrm{rpm}$ at room temperature.

\section{Animals}

Forty-four seven-week-old male Sprague-Dawley rats weighing 200 to $220 \mathrm{~g}$ (Orient Bio Comp., Seongnam, Korea) were used as a rat model for human fat tissue grafts. During the study, the rats were bred under a constant laminar airflow and given standard laboratory chow and water. The rats were used after a twoday adaptation period. The scalp was used as the recipient site for all fat injections [7]. The Animal Care and Use Committee of our institution consented to the experimental protocol.

\section{Streptozotocin-induced diabetes}

The rats were randomly divided into two groups (a diabetic group $[n=24]$ or a control group $[n=20])$. Diabetes was induced by a streptozotocin (STZ) injection. All of the rats were totally fasted for 24 hours before injection. The animals in the diabetic group were injected once with STZ $(60 \mathrm{mg} / \mathrm{kg}$, intraperitoneally, Sigma-Aldrich) dissolved in a citrate buffer $(0.1 \mathrm{~mol} / \mathrm{L}$, $\mathrm{pH} 4.5$, Sigma-Aldrich) [8]. The rats in the control group were injected with an equal volume of the citrate buffer. Diabetes was confirmed as a blood glucose level $>300 \mathrm{mg} / \mathrm{dL}$ at 3 days after the STZ injection [8]. Blood glucose levels were checked using the One Touch Ultra Glucometer (Accu-chek, Roche Bioproducts, Basel, Switzerland) twice weekly.

\section{Fig. 2. The rat scalp after fat injection}

A photograph of the control group rat scalp 10 weeks after fat injection. The hump on the scalp was well maintained and there was no single symptom of immune rejection detected, such as infection signs, redness, edema or necrosis.

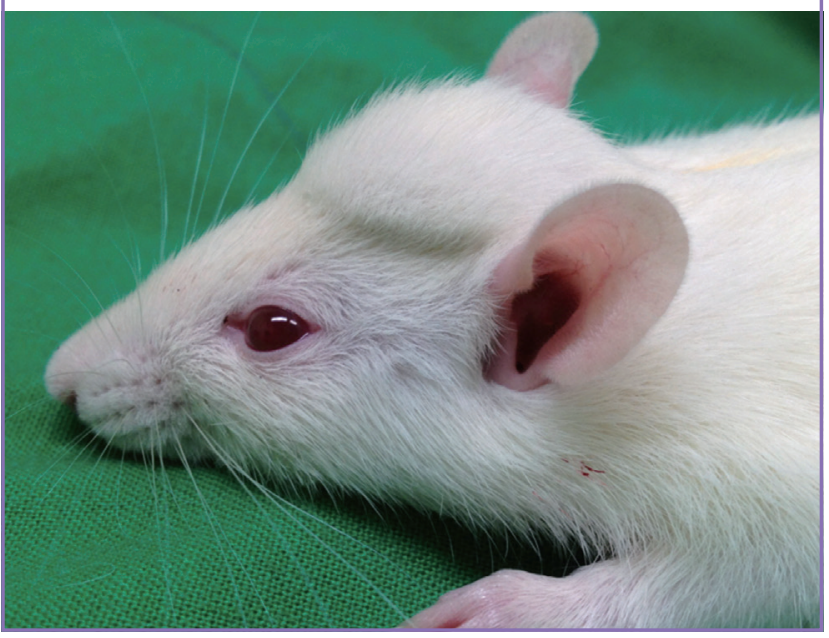




\section{Fat injection}

Processed fats were transferred into $1.0 \mathrm{~mL}$ syringes. Before fat injection, Zoletil (Tiletamine+Zolazepam [1:1], 30 mg/kg; Virbac Korea Inc., Seoul, Korea) and Rompun (xylazine, $10 \mathrm{mg} / \mathrm{kg}$; Bayer Korea Inc., Seoul, Korea) were injected intraperitoneally. After achieving anesthesia, $1.0 \mathrm{~mL}$ of fat was injected subcutaneously into the scalp of each rat with a 16-gauge needle (Fig. 2). No post-procedural analgesics or antibiotics were administered to any animal over the 10-week study period, during which animals were checked daily for clinical signs of immune rejection and infection and for behavioral problems.

\section{Fat graft dissection and analysis}

At the end of the 10-week study period, the rats were euthanized with $\mathrm{CO}_{2}$ gas, the fat pads were excised and their weights and volumes determined; volumes were obtained using the liquid displacement method. In addition, fat grafts were sent for histological examination. In brief, sections of fat pads were stained with hematoxylin and eosin first and inspected under a light microscope. The histology slides were examined for the following parameters: 1) the presence of intact and nucleated lipocytes; 2) the presence of cysts and vacuoles; 3) degree of inflammation, as evidenced by lymphocyte and macrophage infiltration; 4) vascularity; and 5) collagen and elastic fibrils, which indicated the presence of fibrosis and connective tissue components $[1,9]$. Each parameter was scored with a semi quantitative scale ranging from 0 to 5 . Scores were allocated on the basis of the relative presence of the histological parameters $(0$, absent; 1 , minimally; 2 , minimally to moderately; 3 , moderately; 4 , moderately to extensively; and 5, extensively) [1]. All sections were scored independently by three blinded investigators. Further, CD31 (Abcam, ab24590; Cambridge, England) immunostaining was performed to confirm vascularity.

\section{Statistics}

Fat graft weights and volumes were analyzed using the independent samples t-test, and the Mann-Whitney nonparametric test was used for analyzing the ranked histological parameters. The analysis was completed with PASW ver. 18.0 for Windows (SPSS Inc., Chicago, IL, USA), and statistical significance was accepted for P-values $<0.05$.

\section{RESULTS}

\section{Body weights and blood glucose levels}

During the 10-week study period, each rat was weighed weekly and blood glucose levels were checked twice weekly using One Touch Ultra Glucometer by tail punching. At the end of the 10- week study period, the mean body weights in the diabetic and control groups were $298.5 \mathrm{~g}$ and $567.4 \mathrm{~g}$, respectively. Blood glucose levels in the diabetic group were maintained between 300 $\mathrm{mg} / \mathrm{dL}$ and $500 \mathrm{mg} / \mathrm{dL}$ in 21 rats. The other three rats exhibited excessive hyperglycemia (blood glucose $>500 \mathrm{mg} / \mathrm{dL}$ ) and their body weights continuously decreased. One of the three died 1 week post-injection. The other 2 rats died of hyperglycemia 1 month post-injection. We dissected the scalp area to search for immune rejection, but there was no evidence of infection or any immune rejection symptom such as redness, discharge, hemorrhage or necrosis. The rest of the rats were well and survived until the date of euthanasia at 10 weeks after the fat injection.

\section{Gross examination}

$1.0 \mathrm{~mL}$ of processed fat was injected into each rat. The fat graft in the 41 surviving rats remained as a well-defined subcutaneous lump (Fig. 2). No gross evidence of acute inflammation, seroma collection, abscess formation, or tissue necrosis in the grafts or adjacent tissues was observed.

After dissection, grossly all of the fat grafts in the diabetic group rats were half resorbed at 10 weeks post-injection. In contrast, fat grafts in the control group remained intact and appeared as welldefined subcutaneous lumps (Fig. 3).

The average fat graft volumes were $0.413 \mathrm{~mL}$ and $0.745 \mathrm{~mL}$ in the diabetic and control groups, respectively, which was significant $(\mathrm{P}<0.05)$ (Fig. 4$)$, and the average fat graft weights were $0.329 \mathrm{~g}$ and $0.651 \mathrm{~g}$, respectively, which was also significant $(\mathrm{P}<0.05)$

\section{Histological evaluations}

Analysis of the histological findings of hematoxylin and eosinstained specimens revealed significantly more cysts and vacuoles in the diabetic group $(\mathrm{P}<0.05)$. Further, fat grafts were significantly less vascularized and showed significantly more fibrosis in the diabetic group $(\mathrm{P}<0.05)$ (Fig. 5). Although the two groups were not significantly different in terms of the cellular integrity of fat cells and inflammation, diabetic group sections exhibited more inflammation and less fat cell integrity.

The sections of graft taken at 10 weeks were also stained with CD31 antibodies (Fig. 6), and the results obtained showed that fat grafts in the diabetic group were obviously less vascularized.

The final fat volume, weight, and histologic results are compared in Tables 1, 2 .

\section{DISCUSSION}

Fat injection for reconstruction and cosmetic purposes was first described in the late 1980s [10], but it was not until the 1990s, 


\section{Fig. 3. Intra-dissection photographs of the rat scalp}

Intra-dissection photographs of $(A)$ the diabetic group and (B) the control group. Fat grafts in the diabetic group rats showed a half-resorbed state 10 weeks after transplantation. In contrast, the fat grafts from the control group rats were still intact; these grafts existed as welldefined subcutaneous humps.
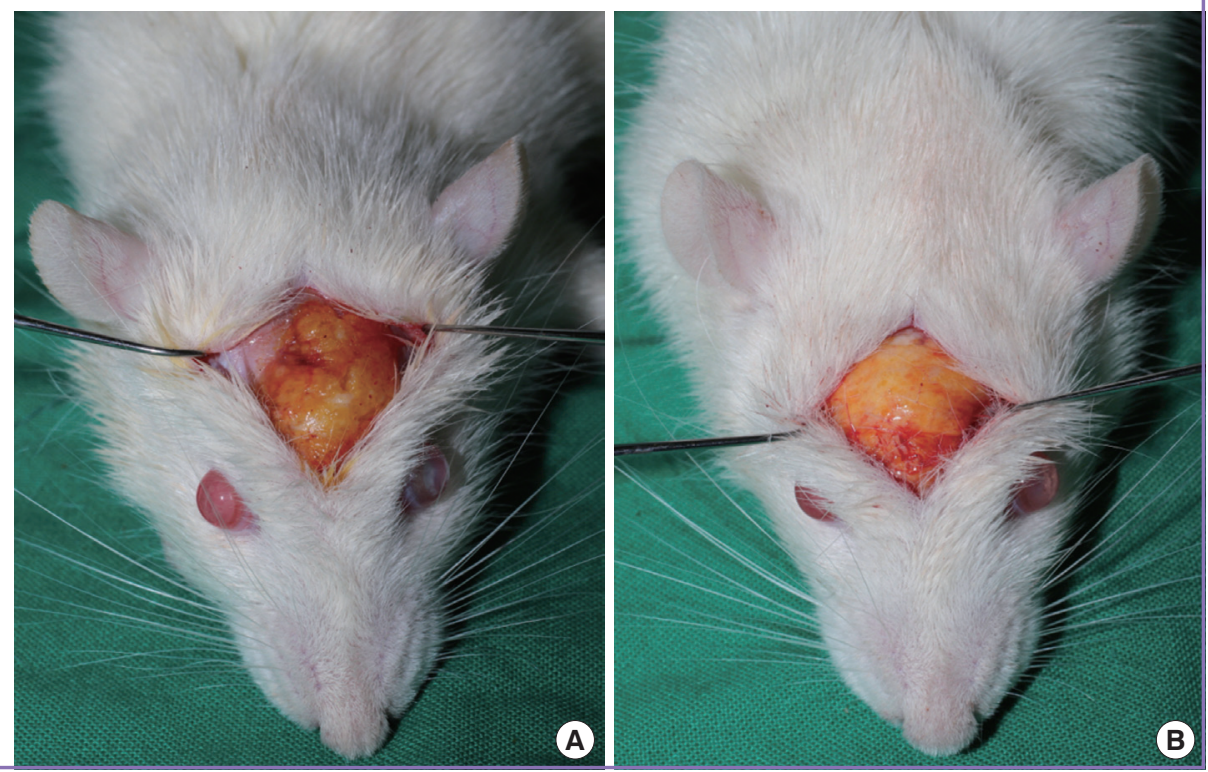

Fig. 4. The dissected fat specimens of two groups

The dissected fat specimens from (A) the diabetic rat and (B) control group.
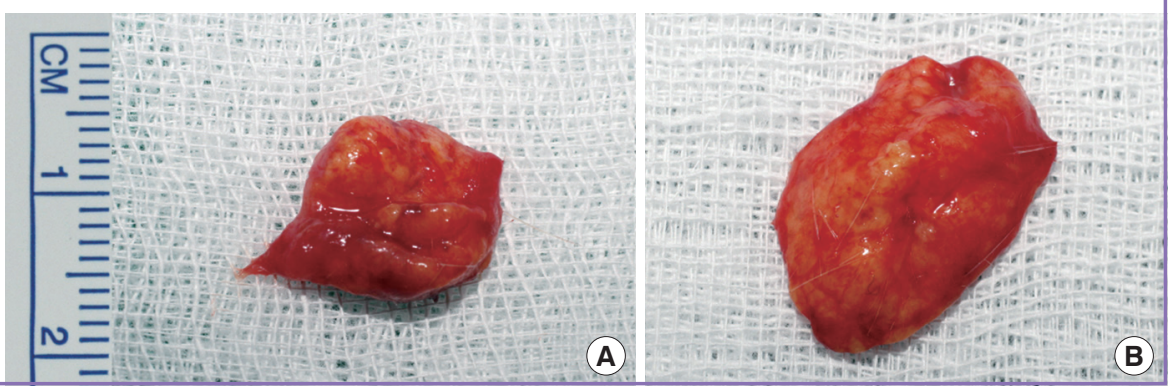

Fig. 5. Histological findings of the diabetic and control groups

Fat specimens from (A) the diabetic group and (B) the control group. Fat grafts were sectioned, stained with H\&E, and imaged with a light microscope $(\times 100)$. Irregularly sized fat cells can be seen in the diabetic group, with multiple cysts, vacuoles, fibrous tissues and inflammatory cells, while evenly sized fat cells are visible in the control groups, with few cysts, vacuoles, and inflammatory cells.

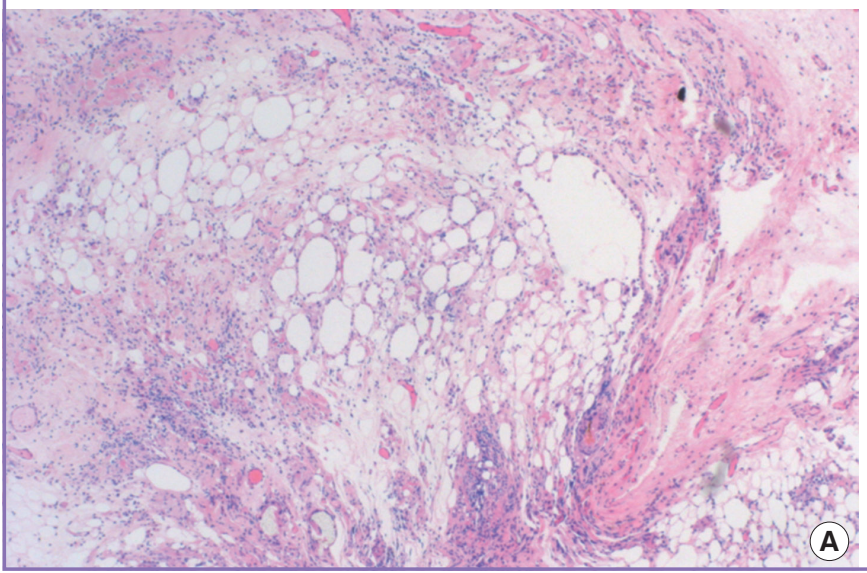

when Coleman [11] outlined a standardized reproducible "process" for fat harvesting, centrifugation, and injection, that the reliability and reproducibility of facial "structural fat grafting" became evident. Subsequently, the use of free autologous fat

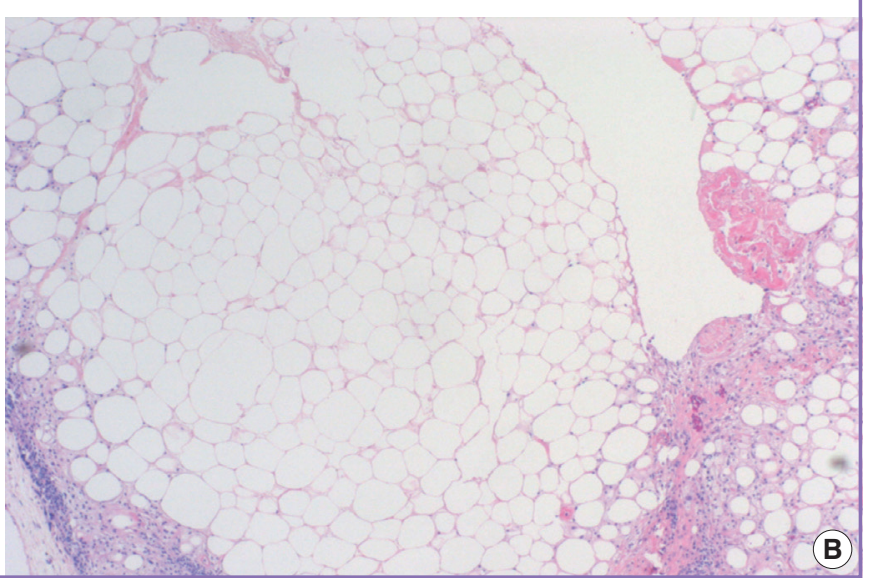

grafts became immensely popular within the medical field, principally because fat tissue is plentiful, easily harvested, and hypoimmunogenic, and also has favorable physical features [12], which include biocompatibility, versatility, a natural appearance, 


\section{Fig. 6. Histological findings of the CD31 antibody stain}

The fat grafts were sectioned, stained with CD31 antibodies, and imaged with a light microscope $(\times 100)$. (A) Histological findings of the diabetic group. (B) Histological findings of the control group. The diabetic group that irregularly sized fat cells exist with vacuoles, fibrous tissues and few vessels surrounded by the intact fat cells. The control group shows evenly sized fat cells with numerous vessels surrounded by intact fat cells. The black arrows indicate vessels. This stain prominently showed that the diabetic group fat graft was less vascularized than the graft in the control group.
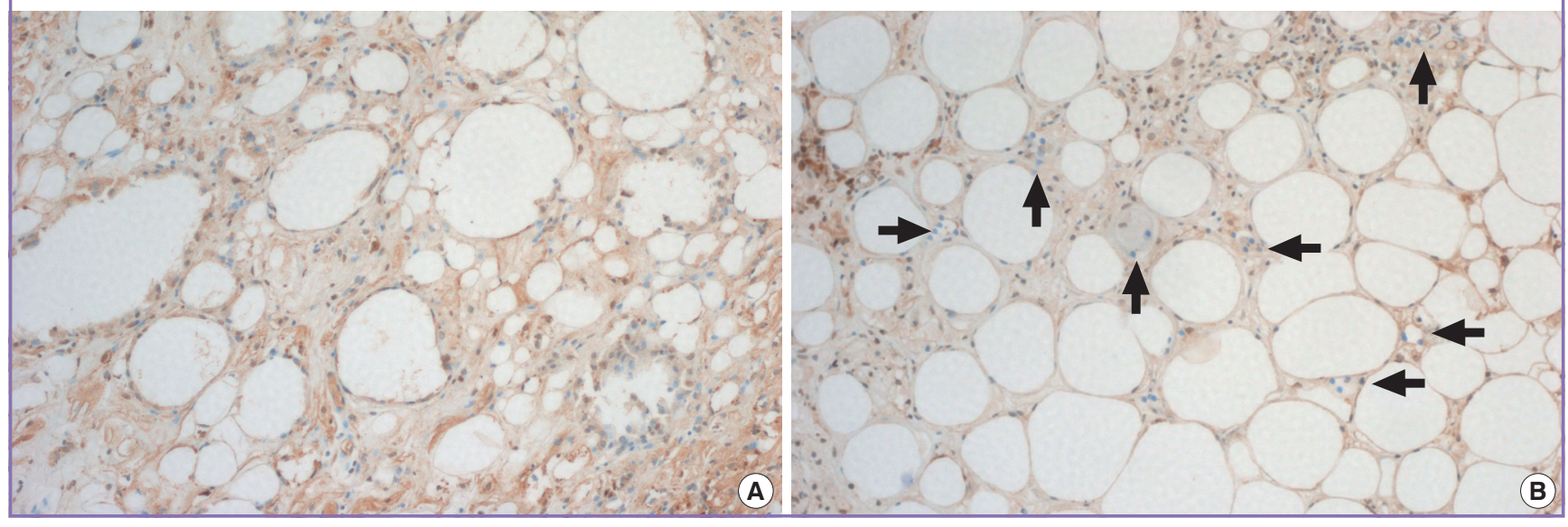

Table 1. Average volume and weight of the fat

\begin{tabular}{|lccc|}
\hline Value & $\begin{array}{c}\text { Diabetic group } \\
(\mathbf{n}=\mathbf{2 1})\end{array}$ & $\begin{array}{c}\text { Control group } \\
(\mathbf{n}=\mathbf{2 0})\end{array}$ & P-value \\
\hline Average volume $(\mathrm{mL})$ & $0.413 \pm 0.125$ & $0.745 \pm 0.182$ & $0.045^{\mathrm{a})}$ \\
Average weight $(\mathrm{g})$ & $0.329 \pm 0.211$ & $0.651 \pm 0.194$ & $0.039^{\mathrm{a})}$ \\
\hline $\begin{array}{l}\text { Statistical analysis: independent samples t-test. } \\
\text { a)Statistically significant }(\mathrm{P}<0.05) .\end{array}$ & \\
\hline
\end{tabular}

and low donor site morbidity. The major drawback of this procedure is the high absorption rate of the injected fat, which is probably the result of ischemia and a lack of neoangiogenesis [13].

Contradictory theories have been proposed regarding volume maintenance after fat grafting [14]. The most commonly established of these theories is Peer's cell survival theory [15]. Damage of the grafted fat is processed immediately during and after injection because of the mechanical injures due to the injection force and because devascularization causes ischemic injury. After the fat injection, on the first day, neutrophils infiltrate the graft, followed by macrophages, histiocytes, and multinucleated giant cells. Histologic examinations of free fat autologous grafts indicate minimal fat cell necrosis at 48 hours post-injection, with only partial graft vascularization. On the fourth day postinjection, from the host bed vascular system, neoangiogenesis leads to graft vascularization. However, the process is slightly limited to the peripheral area of the graft, and the central cells can only obtain marginal access. This process of adipocyte death and removal continues until the reorganized blood supply meets the graft vascular demand, and thus, early and appropriate revascularization is crucial for fat survival. Delays in vascular supply to tissues cause adipocyte necrosis, which include cell membrane
Table 2. Average histological parameter ranks of the fat

\begin{tabular}{|c|c|c|c|}
\hline $\begin{array}{l}\text { Histologic } \\
\text { parameter }\end{array}$ & $\begin{array}{l}\text { Diabetic group } \\
\quad(n=21)\end{array}$ & $\begin{array}{l}\text { Control group } \\
\quad(n=20)\end{array}$ & P-value \\
\hline Integrity & $2.87 \pm 0.69$ & $3.02 \pm 0.91$ & 0.85 \\
\hline Cysts and vacuoles & $3.84 \pm 1.07$ & $2.11 \pm 1.12$ & $0.01^{\text {a) }}$ \\
\hline Inflammation & $1.75 \pm 0.73$ & $1.21 \pm 0.88$ & 0.25 \\
\hline Vascularity & $0.84 \pm 0.33$ & $1.81 \pm 0.75$ & $0.02^{\text {a) }}$ \\
\hline Fibrosis & $2.50 \pm 0.54$ & $1.83 \pm 0.95$ & $0.04^{a)}$ \\
\hline \multicolumn{4}{|c|}{$\begin{array}{l}\text { The values are shown with mean } \pm \text { standard deviation. Statistical analysis: Mann } \\
\text { Whitney nonparametric test. } 0 \text {, absent; } 1 \text {, minimal; } 2 \text {, minimal to moderate; } 3 \\
\text { moderate; } 4 \text {, moderate to extensive; } 5 \text {, extensive. } \\
\text { a)Statistically significant }(P<0.05) \text {. }\end{array}$} \\
\hline
\end{tabular}

detachment and the development of fatty cysts and vacuoles in non-vascularized regions [16]. Thus, vascularization is the most essential factor of graft survival.

Diabetes mellitus is one of the major chronic diseases, and patient numbers continue to increase, as changing lifestyles reduce physical activity and increase obesity. The world prevalence of diabetes among adults between 2010 and 2030 is expected to change as follows: a $20 \%$ increase in the prevalence is expected in developed countries and a $69 \%$ increase in developing countries. Therefore, the number of diabetic aesthetic fat graft candidates is sure to increase.

However, significant proportions of morbidities and mortalities among the diabetic population are due to a slow and poor response to tissue ischemia [17]. When chronic tissue ischemia occurs, poor wound healing results in diabetic ulcers and an increase in the lower extremity amputation rate [18]. On the basis of in vitro evidence of increased levels of apoptosis in highglucose cultures [19] and in cells exposed to hypoxia, it has been assumed that cells exposed to both hyperglycemia and hypoxia 
show synergistic increases in apoptosis that are expected to increase tissue damage [20]. In vitro, one study demonstrated that prolonged incubation of diabetic cells in normal glucose cultures did not reverse this phenotype [17]. In one recent study, diabetic cells were isolated from mice in which diabetes had been induced by streptozocin of 4 weeks duration. This implies that 1 month of diabetes and hyperglycemia can cause cellular changes that cannot be reversed by glucose-level normalization. This also suggests that impaired neovascularization and a broken normal woundhealing mechanism will persist even after diabetes is cured. This glycemic memory has been observed clinically in the context of neovascular damage despite strict prolonged glycemic control [21]. Thus, we suggest that further studies be undertaken to improve neovascularization and reduce resorption rates to broaden the use of fat grafts in diabetic patients for aesthetic purposes or chronic wound reconstruction.

In terms of experimental human fat grafting, almost every previous study has used the athymic nude mouse model because of its resistance to xenograft rejection $[1,2,8,10,22,23]$. However, in the present study, we have focused on the hypo-immunogenic characteristics of fat tissue, and processed fat from a human donor in a traditional maneuver and grafted this into Sprague-Dawley rats. Before performing the main experiment, we performed a pilot study on 5 Sprague-Dawley rats per group to observe an immune rejection response. During the 10 -week pilot experimental period, no symptoms of hyper-acute or acute immune rejection, such as, infection, redness, edema, or necrosis, was detected. The results of the main experiment concurred with the findings of the pilot study. Nevertheless, the authors are planning further studies to determine the reason for the immune tolerance of the grafted human fat.

To summarize, the present study shows that the presence of diabetes impairs the survival and quality of fat grafts as evidenced by the lower fat graft weights and volumes and poorer histologic graft qualities in the diabetic group. These findings caution that if a fat graft is unavoidable in a diabetes patient, the possibility of an unfavorable result should be considered. Finally, we recommend that further studies be undertaken to improve neovascularization and reduce the resorption rate to allow the indications of fat grafting for aesthetic purposes or reconstruction to be widened to include diabetic patients.

\section{REFERENCES}

1. Ramon Y, Shoshani O, Peled IJ, et al. Enhancing the take of injected adipose tissue by a simple method for concentrating fat cells. Plast Reconstr Surg 2005;115:197-201.

2. Langer S, Sinitsina I, Biberthaler P, et al. Revascularization of transplanted adipose tissue: a study in the dorsal skinfold chamber of hamsters. Ann Plast Surg 2002;48:53-9.

3. Karacaoglu E, Kizilkaya E, Cermik H, et al. The role of recipient sites in fat-graft survival: experimental study. Ann Plast Surg 2005;55:63-8.

4. Nguyen A, Pasyk KA, Bouvier TN, et al. Comparative study of survival of autologous adipose tissue taken and transplanted by different techniques. Plast Reconstr Surg 1990; 85:378-86.

5. Shaw JE, Sicree RA, Zimmet PZ. Global estimates of the prevalence of diabetes for 2010 and 2030. Diabetes Res Clin Pract 2010;87:4-14.

6. Capla JM, Grogan RH, Callaghan MJ, et al. Diabetes impairs endothelial progenitor cell-mediated blood vessel formation in response to hypoxia. Plast Reconstr Surg 2007;119:5970.

7. Por YC, Yeow VK, Louri N, et al. Platelet-rich plasma has no effect on increasing free fat graft survival in the nude mouse. J Plast Reconstr Aesthet Surg 2009;62:1030-4.

8. Yang G, Luo C, Yan X, et al. Extracorporeal shock wave treatment improves incisional wound healing in diabetic rats. Tohoku J Exp Med 2011;225:285-92.

9. Oh DS, Cheon YW, Jeon YR, et al. Activated platelet-rich plasma improves fat graft survival in nude mice: a pilot study. Dermatol Surg 2011;37:619-25.

10. Teimourian B. Repair of soft-tissue contour deficit by means of semiliquid fat graft. Plast Reconstr Surg 1986;78:123-4.

11. Coleman SR. Structural fat grafting. Aesthet Surg J 1998; $18: 386,8$.

12. Man D, Plosker H, Winland-Brown JE. The use of autologous platelet-rich plasma (platelet gel) and autologous platelet-poor plasma (fibrin glue) in cosmetic surgery. Plast Reconstr Surg 2001;107:229-37.

13. Locke MB, de Chalain TM. Current practice in autologous fat transplantation: suggested clinical guidelines based on a review of recent literature. Ann Plast Surg 2008;60:98-102.

14. Del Vecchio D, Rohrich RJ. A classification of clinical fat grafting: different problems, different solutions. Plast Reconstr Surg 2012;130:511-22.

15. Peer LA. Cell survival theory versus replacement theory. Plast Reconstr Surg (1946) 1955;16:161-8.

16. Shoshani O, Livne E, Armoni M, et al. The effect of interleukin- 8 on the viability of injected adipose tissue in nude mice. Plast Reconstr Surg 2005; 115:853-9.

17. Jazayeri L, Callaghan MJ, Grogan RH, et al. Diabetes increases p53-mediated apoptosis following ischemia. Plast Reconstr Surg 2008;121:1135-43.

18. Centers for Disease Control and Prevention (CDC). Diabe- 
tes-related amputations of lower extremities in the Medicare population: Minnesota, 1993-1995. MMWR Morb Mortal Wkly Rep 1998;47:649-52.

19. Baumgartner-Parzer SM, Wagner L, Pettermann M, et al. High-glucose: triggered apoptosis in cultured endothelial cells. Diabetes 1995;44:1323-7.

20. Quagliaro L, Piconi L, Assaloni R, et al. Intermittent high glucose enhances apoptosis related to oxidative stress in human umbilical vein endothelial cells: the role of protein kinase $\mathrm{C}$ and NAD(P)H-oxidase activation. Diabetes 2003; 52:2795-804.
21. Retinopathy and nephropathy in patients with type 1 diabetes four years after a trial of intensive therapy. The Diabetes Control and Complications Trial/Epidemiology of Diabetes Interventions and Complications Research Group. N Engl J Med 2000;342:381-9.

22. Shoshani O, Shupak A, Ullmann Y, et al. The effect of hyperbaric oxygenation on the viability of human fat injected into nude mice. Plast Reconstr Surg 2000;106:1390-6.

23. Ullmann Y, Hyams M, Ramon Y, et al. Enhancing the survival of aspirated human fat injected into nude mice. Plast Reconstr Surg 1998;101:1940-4. 